\title{
Pharmacokinetics and pharmacodynamics of intravenous midazolam in patients with severe alcoholic cirrhosis
}

\author{
A J MACGILCHRIST, G G BIRNIE, A COOK, G SCOBIE, T MURRAY, \\ G WATKINSON, AND M J BRODIE
}

From the Departments of Clinical Pharmacology and Gastroenterology, Western Infirmary, Glasgow

SUMMARY Midazolam kinetics and psychomotor function were studied after an intravenous dose of $0.075 \mathrm{mg} / \mathrm{kg}$ body weight in seven patients with alcoholic cirrhosis and eight control patients. Four of the seven cirrhotics died of complications of their liver disease within six months of the study. The metabolism of midazolam was significantly impaired in the cirrhotic patients $(\mathrm{p}<0.025)$. These patients also had evidence of greater sedation than the control group for up to six hours after the dose was administered $(p<0.05)$. The clearance of midazolam did not correlate significantly with the serum albumin, or bilirubin, or with the kinetics of antipyrine, or indocyanine green. This study shows significant delay in the elimination of midazolam and decreased psychomotor function in patients with severe alcoholic liver disease. Caution is needed in using this drug for premedication in such patients before endoscopy.

In chronic liver disease the disposition and elimination of many lipid soluble drugs are altered. ${ }^{12}$ The hepatic metabolism of these agents may be determined by the capacity of the oxidising and conjugating enzyme systems and the liver blood flow, ${ }^{3}$ both of which may be impaired in the presence of cirrhosis. ${ }^{4}$ The pharmacological response to a drug may also be abnormal in liver disease and, in particular, the cerebral sensitivity to sedative drugs may be increased. ${ }^{5}$

Patients with chronic liver disease are frequently referred for upper gastrointestinal endoscopy either for the diagnostic assessment of gastrointestinal haemorrhage or dyspepsia or for therapeutic injection sclerotherapy of oesophageal varices. ${ }^{6}$ Diazepam is commonly used as the premedicating sedative, but in patients with liver disease the half life of this drug may exceed 100 hours, ${ }^{7}$ and some patients exhibit increased cerebral sensitivity. ${ }^{89}$

Midazolam is a new benzodiazepine consisting of an imidazole ring fused at the 1.2 position with a diazepine ring. Its hypnotic action is brief and its elimination half life is under four hours and in some patients is as short as two hours. ${ }^{10-12}$ These properties make it a potentially useful sedative for minor

Address for correspondence: Dr G Birnie, Gardiner Institute, Western Infirmary, Dumbarton Road, Glasgow G11 6NT.

Received for publication 7 May 1985 invasive procedures. It is metabolised in the liver by hydroxylation and conjugation with glucuronic acid. ${ }^{13}$ It has a high hepatic extraction ratio ${ }^{10}$ and so its elimination would be expected to be dependent on liver blood flow.

The aims of this study were to compare the pharmacokinetic profile and pharmacodynamic response after an intravenous dose of midazolam in patients with hepatic cirrhosis and those with normal liver function. Secondly, to assess the value of antipyrine and indocyanine green kinetics in predicting midazolam pharmacokinetics and dynamics.

\section{Methods}

PATIENTS

Seven patients with hepatic cirrhosis and eight patient controls agreed to take part in the study. All gave written informed consent to inclusion in the study which was approved by the local hospital ethical committee.

The cirrhotic patients were aged between 39 and 54 years and all had biopsy proven alcoholic cirrhosis. They were referred for endoscopy for the assessment of portal hypertension. Five of the seven patients had previously bled from oesophageal varices. Two had previous episodes of portosystemic encephalopathy, but there was no clinical evidence 
of this on entry to the study. Four of these patients died within six months of completion of the study from complications of their liver disease.

The patient controls were aged between 37 and 62 years. They showed no clinical evidence of liver disease and had normal biochemical liver function tests. They were all referred for diagnostic upper gastrointestinal endoscopy for suspected peptic ulcer disease.

No patient was receiving any drug known to alter hepatic drug metabolism or liver blood flow and all patients were instructed to abstain from ethanol for one week before the study.

Routine liver function tests (serum albumin, alanine transferase (ALT), aspartate transferase (AST), bilirubin, gamma glutamyl transpeptidase (GGT) and alkaline phosphatase) were obtained by standard autoanalyser techniques (TECHNICON).

After an overnight fast $600 \mathrm{mg}$ of antipyrine was administered orally and $10 \mathrm{ml}$ blood samples were taken at $0,3,5,8,12,24$, and 46 hours. Antipyrine analysis was done using high pressure liquid chromatography. ${ }^{14}$ Indocyanine green was given as an intravenous bolus of $0.5 \mathrm{mg} / \mathrm{kg}$ body weight with the patients fasted and supine. Venous blood samples were taken at three minute intervals to 21 minutes. Venous blood samples were taken at three minute intervals to 21 minutes. Indocyanine green was measured spectrophotometrically. ${ }^{15}$

On the day of the endoscopy the patients were fasted and immediately before the examination an intravenous bolus of $0.075 \mathrm{mg} / \mathrm{kg}$ of midazolam was administered. Venous blood samples were taken from the contralateral arm at $2,5,10,15,20,25,30$, 60,90 minutes and at $2,3,4,6,12$, and 24 hours. Midazolam plasma concentrations were determined using a gas chromatography method. ${ }^{16}$ This assay has a sensitivity of $5 \mathrm{mg} / \mathrm{ml}$, and a six point calibration curve and was included in each sample batch along with quality assurance samples.

Psychomotor function was assessed at 1, 2, 4, 6, 12 , and 24 hours after injection using a Leeds psychomotor tester. This measures the critical flicker fusion threshold (CFFT) and choice reaction time (CRT). ${ }^{17}$ The choice reaction time is divided into two components; firstly, the time taken to move the hand from a switch when a light is switched on (recognition time or CRT 1) and thereafter the time taken to move to the appropriate switch (movement time). The total reaction time (recognition time +movement time) is referred to as the CRT 2. On each occasion a mean of six CFFT and 30 CRT readings were taken. During the 24 hours before receiving midazolam the subjects had a minimum of three practice runs on the Leeds tester followed by three further runs whose results were meaned to produce baseline values for each patient. For the analysis at each time point the difference between the mean CFFT/CRT and the patient's baseline results has been calculated and the mean of these differences for each time point compared between the control and cirrhotic group.

For antipyrine and indocyanine green the pharmacokinetic constant of elimination (K) was calculated by the method of least square regression analysis assuming a one compartment model. The apparent volume of distribution (vd) was calculated from the formula $v d=D O S E / C O$ where $C O=$ the concentration of the drug at time zero extrapolated on a long-linear scale. The apparent plasma clearance (cl) was calculated from the relationship $\mathrm{cl}=\mathrm{vd}^{*} \mathrm{~K}$. For midazolam the plasma concentrations were analysed with a two compartment model using a two compartment logarithmic fit. The results are expressed as means \pm standard error of the mean (SEM) unless otherwise stated.

Statistical analyses were by Mann Whitney U tests and paired Wilcoxon's signed rank tests. The correlation coefficients were calculated using the Spearman ranking procedure.

\section{Results}

All patients became overtly sedated as a result of the administration of midazolam and endoscopy was accomplished with minimal difficulty. The standard biochemical liver function tests were all significantly deranged in the cirrhotic patients (Table 1).

\section{PHARMACOKINETICS}

The elimination of antipyrine and indocyanine green in the two groups is shown in Table 2; there was a highly significant prolongation of the half lives of both drugs in the cirrhotic patients $(p<0 \cdot 001)$, with

Table 1 Clinical details and biochemical liver function tests (mean $+S E M)$ in cirrhotics and controls

\begin{tabular}{lcc}
\hline & $\begin{array}{l}\text { Controls } \\
n=8\end{array}$ & $\begin{array}{l}\text { Cirrhotics } \\
n=7\end{array}$ \\
\hline Age range (yr) & $37-42$ & $39-54$ \\
Weight (kg) & $60 \cdot 6 \pm 4$ & $71 \cdot 3 \pm 4 \cdot 3$ \\
Dose of midazolam (MG) & $4 \cdot 5 \pm 0$ & $5 \cdot 4 \pm 0 \cdot 3$ \\
Bilirubin ( $\mu$ mol/l) & $9 \cdot 1 \pm 0$ & $71 \cdot 7 \pm 35 \cdot 9 \ddagger$ \\
Alkaline phosphatase (IU/l) & $87 \cdot 9 \pm 6$ & $231 \cdot 2 \pm 61 \cdot 0 \ddagger$ \\
Alanine transferase (IU/l) & $23 \cdot 3 \pm 1$ & $60 \cdot 0 \pm 9 \cdot 4 \ddagger$ \\
Aspartate transferase (IU/l) & $22 \cdot 6 \pm 2 \cdot 6$ & $39 \cdot 7 \pm 7 \cdot 4^{*}$ \\
Gamma glutamyl & & \\
$\quad$ transpeptidase (IU/l) & $24 \cdot 9 \pm 3 \cdot 6$ & $164 \cdot 4 \pm 54 \cdot 2 \ddagger$ \\
Albumin (g/l) & $44 \cdot 0 \pm 0 \cdot 9$ & $33 \cdot 0 \pm 2 \cdot 9 *$ \\
\hline
\end{tabular}

Statistics obtained by Mann Whitney $U$ tests.

${ }^{*}=p<0.05 \ddagger=p<0.001$. 
Table 2 Antipyrine, indocyanine green and midazolam kinetics (mean $\pm S E M)$ in cirrhotics and controls

\begin{tabular}{lrc}
\hline & $\begin{array}{l}\text { Controls } \\
n=8\end{array}$ & \multicolumn{1}{l}{$\begin{array}{l}\text { Cirrhotics } \\
n=7\end{array}$} \\
\hline Antipyrine & $9 \cdot 8 \pm 1 \cdot 1$ & $19 \cdot 6 \pm 1 \cdot 6 \ddagger$ \\
Half life (h) & $45 \cdot 5 \pm 4 \cdot 3$ & $60 \cdot 7 \pm 7 \cdot 2$ \\
Volume of distribution (litres) & $1 \cdot 0 \pm 0 \cdot 2$ & $0 \cdot 6 \pm 0 \cdot 1^{*}$ \\
Clearance (ml/min/kg) & & \\
Indocyanine green & $4 \cdot 1 \pm 0 \cdot 3$ & $27 \cdot 9 \pm 8 \cdot 3 \ddagger$ \\
Half life (min) & $4 \cdot 1 \pm 0 \cdot 4$ & $4 \cdot 5 \pm 0 \cdot 3$ \\
Volume of distribution (litres) & $12 \cdot 4 \pm 1 \cdot 4$ & $2 \cdot 6 \pm 0 \cdot 7 \ddagger$ \\
Clearance (ml/min/kg) & & \\
Midazolam§ & $8 \cdot 1 \pm 0 \cdot 6$ & $4 \cdot 8 \pm 0 \cdot 4$ \\
Distribution half life (min) & $1 \cdot 6 \pm 0 \cdot 3$ & $3.9 \pm 0 \cdot 8 \dagger$ \\
Elimination half life (h) & $10 \cdot 4 \pm 1 \cdot 3$ & $5 \cdot 4 \pm 1 \cdot 0 \dagger$ \\
Clearance (ml/min/kg) & $80 \cdot 7 \pm 10 \cdot 3$ & $106 \cdot 19 \pm 11 \cdot 9$ \\
Volume of distribution (litres) & 8.9 \\
\hline
\end{tabular}

Statistics obtained by Mann Whitney U tests.

$\dot{p}=<0.05 ; \dagger=p<0.025 ; \ddagger=p<0.001 ; \S$ Cirrhotics $n=5$.

a concomitant reduction in the clearance $(p<0.05$ and $\mathrm{p}<0.001$ ).

The metabolism of midazolam was impaired in the cirrhotic group compared with controls. In two patients ( $A$ and $B$ in Figure 1) high plasma concentrations of midazolam were found throughout the duration of the study. If the values are true there the greatly prolonged estimated half life would preclude the calculation of acceptable kinetic parameters. Another possibility is that these results are because of some substance interfering with the assay, but attempts have failed to identify an interfering peak. The analytical data from these patients are therefore plotted separately and are not included in the statistical analysis of the midazolam kinetics. The distribution half life of midazolam was slightly reduced in the cirrhotic patients compared to the controls (cirrhosis $=4.58 \pm 1.83$ mins $v s$ controls $=6.44 \pm 1.08 \mathrm{~min}$ ) but this difference did not reach statistical significance. The elimination half lives were significantly prolonged in the cirrhotic group (cirrhosis $=2.89 \pm 1.08$ hours $v s$ controls $=1.65 \pm 0.26: p<0.025)$ and the clearances were appropriately reduced (cirrhosis $=5.67 \pm 0.99 \mathrm{ml} /$ $\mathrm{min} / \mathrm{kg} v s$ controls $=10.62 \mathrm{ml} / \mathrm{min} / \mathrm{kg}: \mathrm{p}<0.025$ ).

There were no significant correlations between the clearance of midazolam with the serum albumin, serum bilirubin, or with the clearances or half lives of antipyrine or indocyanine green.

PHARMACODYNAMICS

All the control and cirrhotic patients were included in these analyses.

\section{Choice reaction time}

The CRT 1 (recognition time) and CRT 2 (total

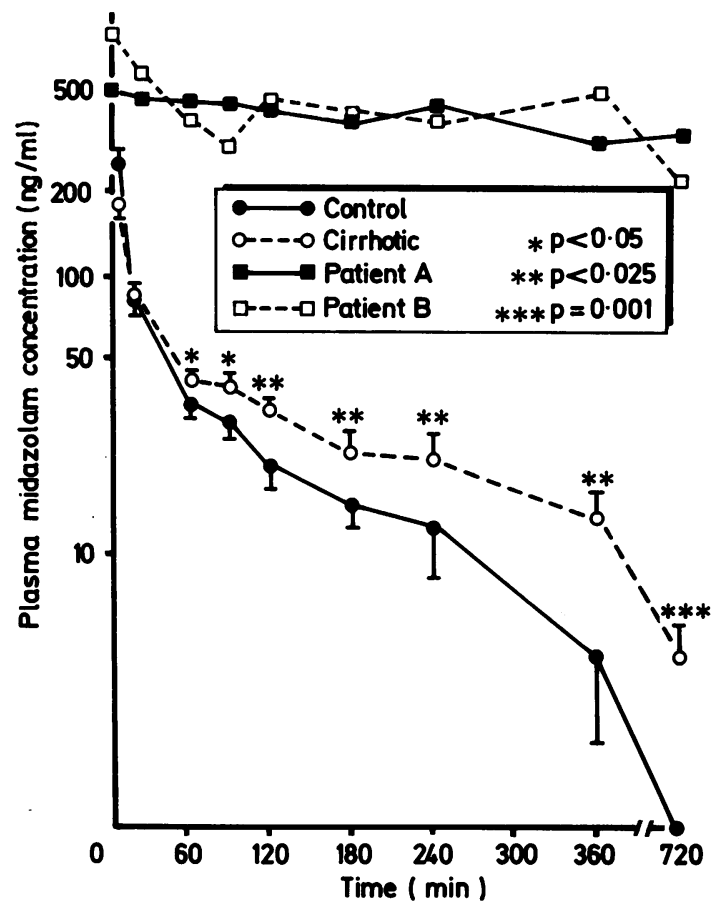

Fig. 1 Midazolam plasma concentrations (mean $\pm S E M$ ) in eight control and five cirrhotic patients after an intravenous dose of $0.075 \mathrm{mg} / \mathrm{kg}$ body weight. Actual plasma concentrations are plotted for patients $A$ and $B$. Statistical analysis by Mann Whitney U tests.

reaction time) values for both groups are compared in Figures 2 and 3 . There was no significant difference in the mean baseline CRT 1 or CRT 2 between the cirrhotic and control groups. Neither the CRT 1 nor CRT 2 were significantly prolonged in the control patients at any time during the course of the study. In the cirrhotic patients both the CRT 1 and CRT 2 were prolonged compared with the baseline values at all time points after the administration of midazolam and these differences were statistically significant at one, four and six hours $(p<0.025)$. Comparison of the cirrhotic and control patients showed significant prolongation of the CRT 1 at one hour $(\mathrm{p}<0.025)$, four hours $(\mathrm{p}<0.05)$ and six hours $(\mathrm{p}<0.025)$, and of the CRT 2 at one, two, and six hours ( $\mathrm{p}<0.025$ at all points) in the cirrhotic group ( 2 tailed Mann Whitney U test).

\section{Critical flicker fusion time}

Critical flicker fusion time values for both groups are shown in Figure 4. The baseline values in the cirrhotic patients were significantly lower than in the 


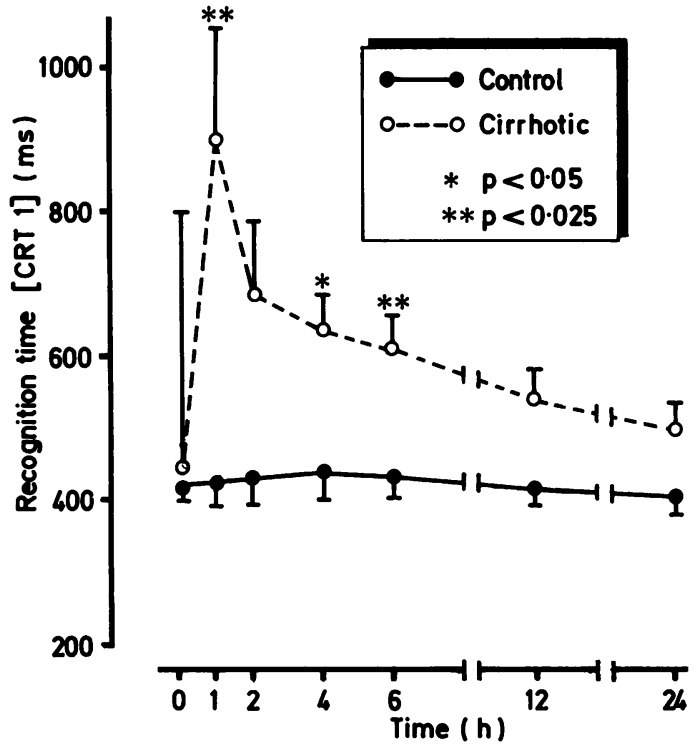

Fig. 2 Recognition time (CRT 1; mean \pm SEM) in eight control patients and seven cirrhotic patients after an intravenous bolus of $0.075 \mathrm{mg} / \mathrm{kg}$ of midazolam. Statistical analysis by Mann Whitney U tests comparing the difference of the results and the baselines in each group.

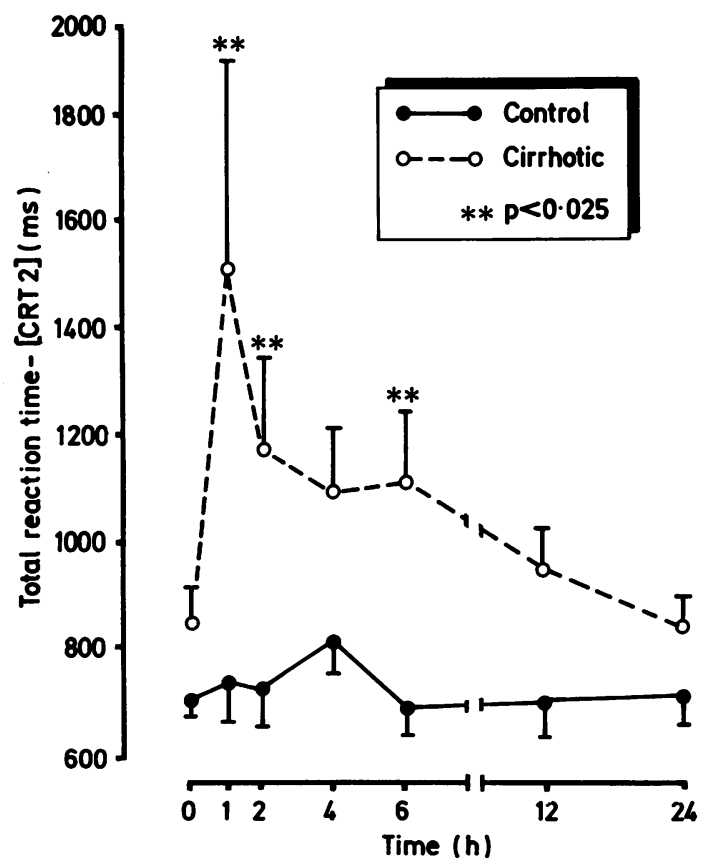

Fig. 3 Total reaction time (CRT 2; mean $\pm S E M)$ in eight control patients and seven cirrhotic patients after an intravenous bolus of $0.075 \mathrm{mg} / \mathrm{kg}$ of midazolam. Statistical analysis by Mann Whitney $U$ tests comparing the difference of the results and the baselines between each group.

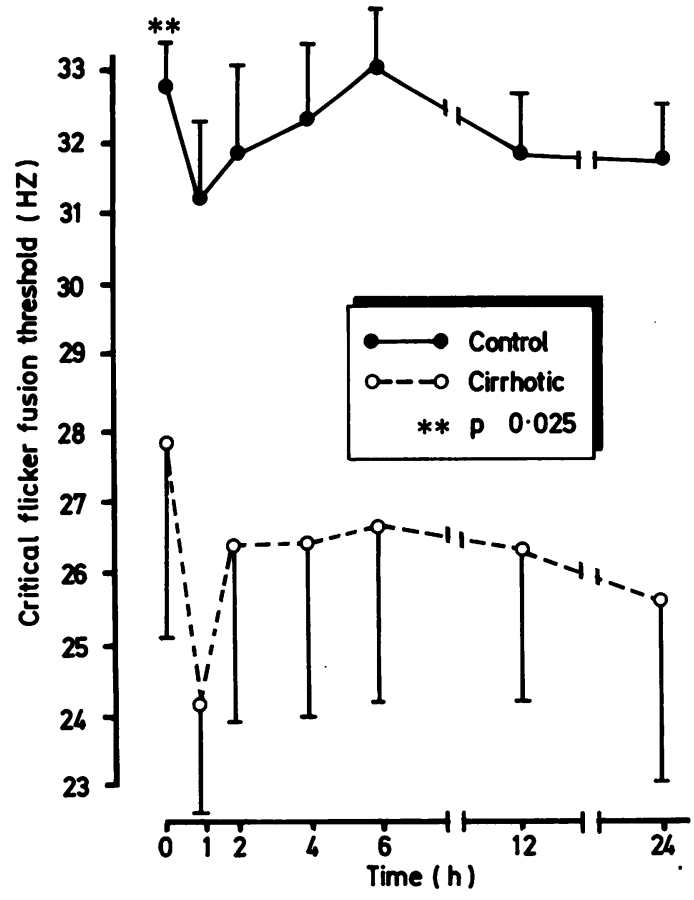

Fig. 4 Critical flicker fusion threshold (CFFT; mean $+S E M)$ in eight control patients and seven cirrhotic patients after an intravenous bolus of $0.075 \mathrm{mg} / \mathrm{kg}$ of midazolam. Statistical analysis by Mann Whitney U test on the comparisons with the baseline results for each group. The baseline differed significantly $(p<0.025)$ between the groups.

control group $(\mathrm{p}<0.05)$. In the control group there was a reduction in the threshold at 60 minutes $(p<0.05)$ after which the threshold returned to normal. In the cirrhotic patients the threshold was significantly reduced at 60,120 and 240 minutes $(\mathrm{p}<0.025)$. Direct comparison between the groups was not done because of the differing baseline values.

\section{Discussion}

This study has shown altered pharmacokinetics of midazolam in patients with chronic liver disease. There was a significant prolongation of the elimination half life of the drug with a concomitant fall in its clearance. The two patients with apparently high concentrations remain unexplained. If these are true concentrations the patients did not appear to be unduly sedated. Similar high concentrations have been shown in critically ill patients receiving repeated doses of midazolam ${ }^{18} 19$

The only objective evidence of psychomotor 
impairment in the control patients was a reduced CFFT at one hour. Subjectively they were sedated sufficiently to allow endoscopy to be carried out. Using the recognition time and the total reaction time there was objective evidence of psychomotor impairment in the cirrhotic patients which was significant for up to six hours after the administration of midazolam. The maximum prolongation in CRT occurred in the cirrhotics at two hours (Figs. 2 and 3). This occurred when there was a small but significantly higher plasma midazolam than in the control patients. These results could be interpreted to suggest that there may be increased cerebral sensitivity to midazolam in these patients with chronic liver disease.

The results of the critical flicker fusion threshold are difficult to interpret because of the significantly reduced baseline in the cirrhotic patients (this problem has also occurred in a similarly designed study, with another psychoactive agent ${ }^{20}$ ). If this apparent initial abnormality represents subclinical portosystemic encephalopathy then the CFFT merits further study as a possible simple means of identifying and monitoring the early stages of this important complication.

The effect of chronic liver disease on the pharmacokinetics and pharmacodynamics of the benzodiazepines varies between the drugs. ${ }^{21}$ The elimination half life of diazepam is prolonged in cirrhotics, mainly because of a reduction in the volume of distribution. ${ }^{9}$ Similarly Kraus et $a l^{22}$ reported a reduced elimination half life of lorazepam attributable to a fall in the volume of distribution. Chlordiazepoxide has a low intrinsic clearance and a high level of protein binding. In cirrhosis both are reduced and the volume of distribution is increased producing a half life which is markedly prolonged. ${ }^{23}$ The elimination of oxazepam, a conjugated benzodiazepine, appears to be unaltered in both acute viral hepatitis or in cirrhosis. ${ }^{24}$ Direct comparisons of midazolam and diazepam in patients without liver disease undergoing diagnostic endoscopy suggest that midazolam produces a more rapid onset of sedation. The degree of sedation is similar and the patient recall is less. ${ }^{25} 26$ This study shows a significant delay in the elimination of midazolam and increase in the degree of sedation produced for up to six hours after its administration to patients with severe alcoholic liver disease. Caution should therefore be exercised in administering the drug to patients with chronic liver disease. These changes, however, may be less than those occurring with diazepam and midazolam may prove to be the benzodiazepine of choice in providing premedication for such patients who require to undergo a diagnostic or therapeutic endoscopic procedure.
We are grateful to Roche Products Ltd for their support and assistance with this project.

\section{References}

1 Hoyampa AM, Branch RA, Schenker S. The disposition and effects of sedatives and analgesics in liver disease. Ann Rev Med 1978; 29: 205-18.

2 Williams RL. Drug administration in hepatic disease. $N$ Engl J Med 1983; 309: 1616-22.

3 Rowland M, Benet LZ, Graham GG. Clearance concepts in pharmacokinetics. J Pharmacokin Biopharm 1973; 1: 123-36.

4 Wilkinson GR, Shand DG. A physiological approach to hepatic drug clearance. Clin Pharm Ther 1976; 18: 377-90.

5 Read AE, Laidlaw J, McCarthy CF. The effects of chlorpromazine in patients with hepatic disease. $\mathrm{Br}$ Med J 1969; 3: 497-9.

6 MacDougall BRD, Westaby D, Theodossi A, Dawson $\mathrm{JL}$, Williams $\mathrm{R}$. Increased long-term survival in variceal haemorrhage using injection sclerotherapy. Lancet 1982; 1: 124-7.

7 Klotz U, Avant GR, Hoyumpa A, Schenker S, Wilkinson GR. The effects of age and liver disease on the disposition and elimination of diazepam in adult man. J Clin Invest 1975; 55: 347-59.

8 McConnell JB, Curry SH, Davies M, Williams R. Clinical effects and metabolism of diazepam in patients with chronic liver disease. Clin Sci 1982; 63: 75-80.

9 Branch RA, Morgan MH, James J, Read AE. Intravenous administration of diazepam in patients with chronic liver disease. Gut 1976; 17: 975-83.

10 Allonen H, Zeigler G, Klotz U. Midazolam kinetics. Clin Pharm Ther 1981; 30: 653-61.

11 Greenblatt DJ, Locniskar A, Ochs HR, Lauven PM. Automated gas chromatography for studies of midazolam pharmacokinetics. Anaesthesiology 1981; 55: 176-9.

12 Smith MT, Eadie MJ, Brophy TOR. The pharmacokinetics of midazolam in man. Eur J Clin Pharmacol 1981; 19: 271-8.

13 Heizmann P, Ziegler WH. Excretion and metabolism of 14C-midazolam in humans following oral dosing. Arzneimittelforsch 1981; 31: 2220-3.

14 MacPhee GJA, Thomson GG, Scobie G et al. Effect of cimetidine on carbamazepine auto- and hetro-induction in man. Br J Clin Pharmacol 1984; 18: 411-9.

15 Caesar J, Shaldon S, Chiandussi L, Guevara L, Sherlock $S$. The use of indocyanine green in the measurement of hepatic blood flow and as a test of hepatic function. Clin Sci 1961; 21: 43-57.

16 Heizmann P, Von Alten R. Determination of midazolam and its alpha hydroxymetabolite in plasma by gas chromatography and electron capture detection. J High Resol Chromatogr Com 1981; 4: 266-9.

17 Hindmarch I. Psychomotor function and psychoactive drugs. Br J Clin Pharmacol 1980; 10: 189-209.

18 Byatt CM, Lewis LD, Dawling S, Cochrane GM. 
Accumulation of midazolam after repeated dosage in patients receiving mechanical ventilation in an intensive care unit. Br Med J 1984; 289: 799-800.

19 Byrne AJ, Yeoman PM, Mace P. Accumulation of midazolam in patients receiving mechanical ventilation. [Letter]. Br Med J 1984; 289: 1309.

20 Parker G, Roberts CJC. Plasma concentrations and central nervous system effects of the new hypnotic agent zopiclone in patients with chronic liver disease. Br J Clin Pharmacol 1983; 16: 259-65.

21 Williams RL, Mamelok RD. Hepatic disease and drug pharmacokinetics. Clin Pharmacokinet 1980; 5: 528-47.

22 Kraus JW, Desmond PV, Marshall JP, Johnson RF, Schenker S, Wilkinson GR. Effects of ageing and liver disease on dispossiition of lorazepam. Clin Pharm Ther 1978; 24: 411-9.
23 Roberts RK, Wilkinson GR, Branch RA, Schenker S. Effect of age and cirrhosis on the disposition and elimination of chlordiazepoxide. [Abstract]. Gastroenterology 1977; 73: A45 1243.

24 Shull HJ, Wilkinson GR, Johnson R, Schenker S. Normal disposition of oxazepam in acute viral hepatitis and cirrhosis. Ann Intern Med 1976; 84: 420-5.

25 Cole SG, Brozinsky S, Isenberg JI. Midazolam, a new more potent benzodiazepine, compared with diazepam: a randomized, double-blind study of preendoscopic sedatives. Gastrointest Endosco 1983; 29: 219-22.

26 Whitwam JG, Al-Khudhairi D, McCloy RF. Comparison of midazolam and diazepam in doses of comparable potency during gastroscopy. Br J Anaesthiol 1983; 55: $773-6$. 